\title{
A novel temperature-responsive micelle for enhancing combination therapy
}

\author{
This article was published in the following Dove Press journal: \\ International Journal of Nanomedicine \\ 25 July 2016 \\ Number of times this article has been viewed
}

\author{
Cheng-Liang Peng ${ }^{1, *}$ \\ Yuan-I Chen $2,3, *$ \\ Hung-Jen Liư ${ }^{2}$ \\ Pei-Chi Lee ${ }^{2}$ \\ Tsai-Yueh Luo' \\ Ming-Jium Shieh ${ }^{2,3}$ \\ 'Isotope Application Division, Institute \\ of Nuclear Energy Research, Taoyuan, \\ ${ }^{2}$ Institute of Biomedical Engineering, \\ College of Medicine and College \\ of Engineering, National Taiwan \\ University, Taipei, ${ }^{3}$ Department of \\ Oncology, National Taiwan University \\ Hospital and College of Medicine, \\ Taipei, Taiwan \\ *These authors contributed equally
} to this work

\begin{abstract}
A novel thermosensitive polymer p(N-isopropylacrylamide-co-poly[ethylene glycol] methyl ether acrylate)-block-poly(epsilon-caprolactone), p(NIPAAM-co-PEGMEA)-b-PCL, was synthesized and developed as nanomicelles. The hydrophobic heat shock protein 90 inhibitor 17-allylamino-17-demethoxygeldanamycin and the photosensitizer cyanine dye infrared-780 were loaded into the core of the micelles to achieve both chemotherapy and photothermal therapy simultaneously at the tumor site. The release of the drug could be controlled by varying the temperature due to the thermosensitive nature of the micelles. The micelles were less than $200 \mathrm{~nm}$ in size, and the drug encapsulation efficiency was $>50 \%$. The critical micelle concentrations were small enough to allow micelle stability upon dilution. Data from cell viability and animal experiments indicate that this combination treatment using photothermal therapy with chemotherapy had synergistic effects while decreasing side effects.
\end{abstract}

Keywords: thermosensitive, photothermal therapy, chemotherapy, nanocarrier, control release, synergistic effect

\section{Introduction}

Nanomedicine ${ }^{1}$ has been widely used in recent years. Many types of nanosized carriers have been designed, including polymeric micelles, ${ }^{2}$ liposomes, ${ }^{3}$ nanoparticles, and drug delivery systems for cancer therapy. ${ }^{4}$ Nanocarriers for drug encapsulation are advantageous for cancer therapy because of preferential accumulation of the drug in the tumors through enhanced permeability and retention. Cancer cells secrete proangiogenic factors that induce angiogenesis at the tumor site, increasing the permeability and pore size of the vessels. Thus, the micelles localized at tumor sites in vivo can enhance the efficiency of treatment and decrease the side effects via enhancing permeability and retention.,

Several studies have demonstrated the destruction of cancer cells by noninvasive photothermal therapy (PTT). ${ }^{7-9}$ Upon absorption of light of a specific wavelength, photosensitive agents (exogenous chromophores, gold nanoparticles, carbon nanotubes, graphene oxide, and organic chromophores such as cyanine dye IR-780) bound to a tumor site facilitate the conversion of light into heat. ${ }^{8}$ PTT thereby increases the temperature of the tissue to over $43^{\circ} \mathrm{C}$, killing the cancer cells. ${ }^{8}$ The organic chromophore IR-780 is a demonstrated PTT agent that is excited by near-infrared wavelengths, increasing the depth of tumor treatment. However, the hydrophobic characteristic of IR-780 to normal cells may limit its clinical use. The encapsulation of IR-780 into the micelles is a possible way to overcome this problem.

PTT can induce thermotolerance in cancer cells through the heat-shock response mediated by several heat-shock proteins (HSPs), including HSP-27, -72, and -90. ${ }^{10} \mathrm{HSPs}$ 
not only lead to resistance in tumors but also reduce the efficiency of PTT. Hence, combined therapies that both enhance the effect of PTT and decrease HSP-induced resistance to chemotherapy are essential. Among the HSP-90 inhibitors used as chemotherapeutic agents, ${ }^{10,11}$ 17-allylamino-17demethoxygeldanamycin $(17-\mathrm{AAG})^{12}$ is the first identified HSP-90 molecular chaperone inhibitor. This agent induces the death of human colon cancer cells in vitro and in vivo ${ }^{13,14}$ but has poor aqueous solubility and serious hepatotoxicity. Increasing the bioavailability and eliminating the toxicity by encapsulating it in the micelles is feasible. ${ }^{14-17}$

In this study, we synthesized a novel thermosensitive amphiphilic polymer ${ }^{18} \mathrm{p}(\mathrm{N}$-isopropylacrylamideco-poly[ethylene glycol] methyl ether acrylate)block-poly(epsilon-caprolactone), p(NIPAAM-coPEGMEA)-b-PCL, by two-step polymerization, which is biocompatible and degradable. ${ }^{19,20}$ With self-assembled thermosensitive micelles, IR-780/17-AAG-coloaded micelles can be employed to effectively administer both PTT and chemotherapy. The in vitro and in vivo experiments were evaluated in human colorectal adenocarcinoma (HCT-116) cell lines. Due to the thermosensitive characteristic of micelle, it demonstrated controlled drug release and subsequent expression during laser irradiation and change of temperature. The development of new IR-780/17-AAG-coloaded micelles should achieve multiple functions including PTT and chemotherapy at the same time, enhancing the ability of killing cancer cells synergistically, as illustrated in Figure 1.

\section{Materials and methods Materials}

Azobisisobutyronitrile (AIBN) was purchased from TCI (Tokyo, Japan). \&-caprolactone was purchased from Acros Organics, Geel, Belgium. Poly(ethylene glycol) methyl ether acrylate (PEGMEA) (MW =480 g/mol), N-isopropylacrylamide (NIPAAM), IR-780 iodide, and 4-cyano-4-(phenylcarbonothioylthio) pentanoic acid (CPPA) were purchased from Sigma-Aldrich, Co., St Louis, MO, USA. Tin(II) 2-ethyleneoctoate $\left(\mathrm{Sn}[\mathrm{Oct}]_{2}\right)$ was purchased from Alfa Aesar (Ward Hill, MA, USA). The anticancer drug 17-AAG (Tanespimycin) was purchased from LC Laboratories (Woburn, MA, USA). All the drugs were used as received. All solvents, including tetrahydrofuran (THF), toluene, dimethylsulfoxide (DMSO), methanol, and ethanol were of high-performance liquid chromatography (HPLC) grade and partly predried over calcium hydride before reaction.

\section{Synthesis of copolymer CPPA-PCL (macro-CPPA) by ring opening polymerization}

The copolymer CPPA-poly( $\varepsilon$-caprolactone) (PCL) (macroCPPA) was synthesized by free-radical polymerization starting from the chain-transfer agent CPPA. ${ }^{21}$ In brief, CPPA (50 mg) was added into a three-necked round-bottom flask and the residual water and oxygen were removed by evacuating and purging with nitrogen for at least 1 hour.

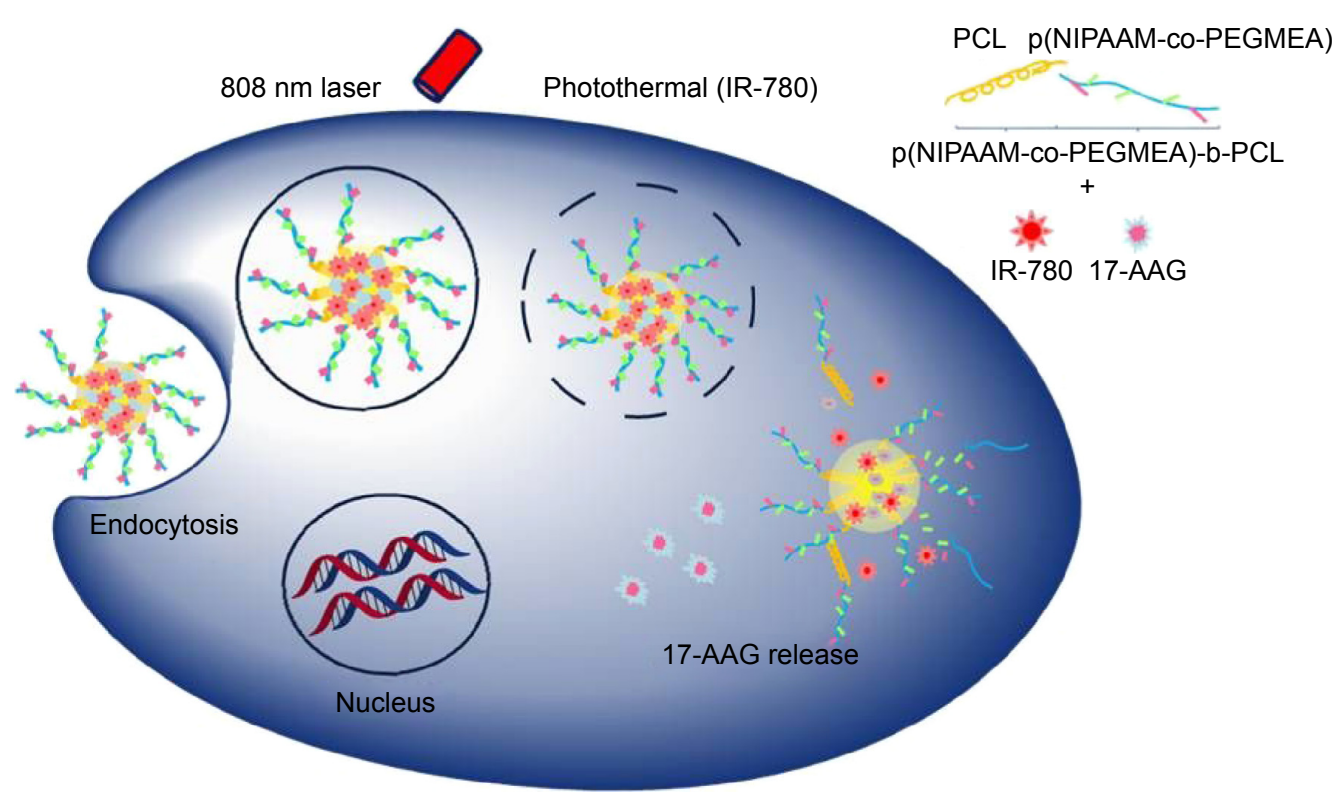

Figure I Thermosensitive micelles encapsulating I7-AAG and IR-780 for temperature-controlled combination therapy.

Abbreviations: I7-AAG, I7-allylamino-17-demethoxygeldanamycin; NIPAAM, N-isopropylacrylamide; PEGMEA, poly(ethylene glycol) methyl ether acrylate; $\mathrm{PCL}$, poly(epsilon-caprolactone); IR, infrared. 


\section{Macro-CPPA by ring opening polymerization}

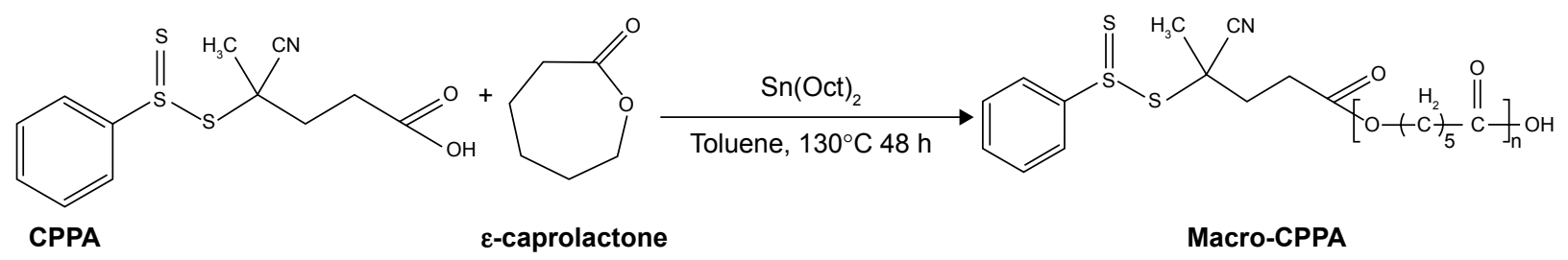

\section{p(NIPAAM-co-PEGMEA)-b-PCL by RAFT polymerization}

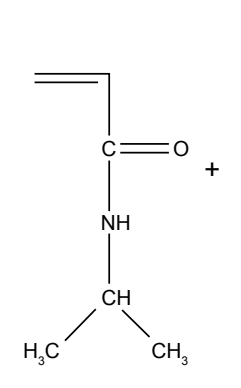

NIPAAM

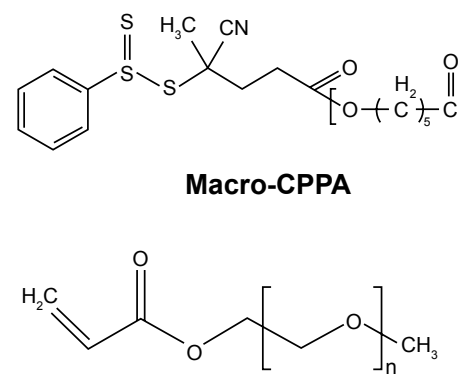

PEGMEA

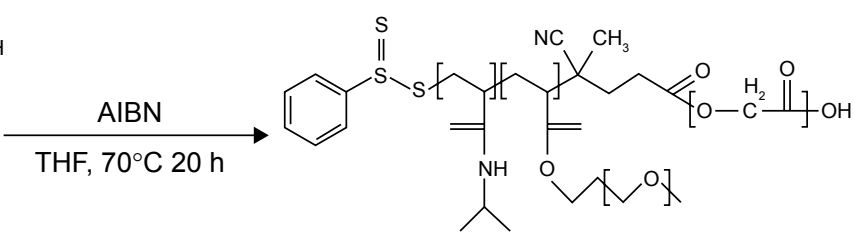

p(NIPAAM-co-PEGMEA)-b-PCL

Figure 2 Synthesis of the copolymer p(NIPAAM-co-PEGMEA)-b-PCL.

Abbreviations: NIPAAM, N-isopropylacrylamide; PEGMEA, poly(ethylene glycol) methyl ether acrylate; PCL, poly(epsilon-caprolactone); RAFT, reversible additionfragmentation chain transfer; CPPA, 4-cyano-4-(phenylcarbonothioylthio) pentanoic acid; THF, tetrahedrofuran; AIBN, azobisisobutyronitrile.

Toluene (20-30 $\mathrm{mL})$, predried over calcium hydride, was then added. A given concentration of $\varepsilon$-caprolactone monomers $(2.2 \mathrm{~mL})$ was added until the reagent was dissolved in the solvent in the presence of $\mathrm{Sn}(\mathrm{Oct})_{2}$ as a catalyst. The reaction was maintained at $130^{\circ} \mathrm{C}$ with continuous stirring for 48 hours under nitrogen. Macro-CPPA powders were precipitated with cold ethyl ether twice to remove unreacted $\varepsilon$-caprolactone monomers, and the remaining products were collected by vacuum filtration. The orange powder obtained was dried under vacuum at room temperature to evaporate the solvent. The details of the method are presented in Figure 2.

\section{Synthesis of $p$ (NIPAAM-co-PEGMEA)-b- $P C L$ by reversible addition-fragmentation chain-transfer polymerization}

NIPAAM (3 g), PEGMEA (2.4 mL), macro-CPPA, and AIBN were dissolved in THF $(40-50 \mathrm{~mL})$ in a $100 \mathrm{~mL}$ three-necked round-bottom flask. The flask was purged with nitrogen for an hour prior to being immersed in a preheated sand bath at $70^{\circ} \mathrm{C}$. Reversible additionfragmentation chain-transfer (RAFT) polymerization was carried out in the flask for 20 hours. The mixtures were then precipitated with cold ethyl ether twice and vacuum-dried overnight. The details of the method are presented in Figure 2.

\section{Polymer characterization}

The ${ }^{1} \mathrm{H}$-nuclear magnetic resonance (NMR) spectrum of macro-CPPA and p(NIPAAM-co-PEGMEA)-b-PCL were determined using a Bruker Avance-500MHz FT-NMR spectrometer (Billerica, MA, USA) with deuterated chloroform $\left(\mathrm{CDCl}_{3}\right)$ as the solvent. Gel permeation chromatography (GPC) was carried out using a Waters 510 HPLC pump (Waters Corporation, Milford, MA, USA) equipped with a 410 differential refractometer. All samples were quantified by GPC using monodispersed polystyrenes as standards using Empower chromatography software (Waters Corporation).

\section{Preparation and characterization of the micelles}

p(NIPAAM-co-PEGMEA)-b-PCL micelles (empty) were prepared as follows. The polymer $(5 \mathrm{mg})$ was dissolved in an appropriate amount of acetone and slowly added drop-wise to $5 \mathrm{~mL}$ phosphate buffer saline (PBS). The residual acetone was eliminated by evaporation under ambient temperature overnight, and the micelles self-assembled.

The IR-780/17-AAG-loaded micelle was prepared using the oil-in-water emulsion solvent evaporation method. ${ }^{22}$ Briefly, IR-780, 17-AAG, and the polymer were dissolved in $1.5 \mathrm{~mL}$ acetone and then slowly added drop-wise to PBS. The ratio of the drug (IR-780 or 17-AAG) to the polymer was 1:6. The residual acetone was eliminated by evaporation under 
ambient temperature overnight, and the drug-encapsulated micelles self-assembled. The solution was then filtered through a $0.45 \mathrm{~mm}$ polyether-sulfone membrane filter (EMD Millipore, Billerica, MA, USA) to remove free drugs and polymer aggregates (Figure S1). The fraction of IR-780loaded micelles was determined by fluorescence with an excitation of $690 \mathrm{~nm}$ and emission of $780 \mathrm{~nm}$. IR-780-loaded micelle solution was added to DMSO (final micelle solution $10 \%, \mathrm{v} / \mathrm{v})$, and fluorescence was detected using a spectrofluorometer (SpectraMax Gemini XS; Molecular Devices, Sunnyvale, CA, USA) or enzyme-linked immunosorbent assay (ELISA) microplate reader (SpectraMax M2 Multimode Microplate Reader, Molecular Devices). In addition, the fraction of 17-AAG-loaded micelles was determined by measuring the absorbance at $332 \mathrm{~nm}$. Using free IR-780 or 17-AAG in DMSO under the same conditions as standards, the drug encapsulation efficiency (EE) and drug loading content (DL) were calculated as follows:

$$
\text { Drug EE }(\%)=\frac{\text { Weight of drug in the micelles }}{\text { Weight of the feeding drug }} \times 100 \%
$$

$\operatorname{DL}(\%)=\frac{\text { Weight of drug }}{\text { Weight of drug }+ \text { Weight of polymer }} \times 100 \%$

The particle mean diameters, polydispersity indexes (PDIs), and zeta potentials of the micelles were measured by dynamic light scattering (DLS) at $25^{\circ} \mathrm{C}$ with a scattering angle of $90^{\circ}$ using a Zetasizer Nano-ZS90 (Malvern Instruments, Malvern, UK).

The morphology of the micelles was observed by transmission electron microscopy (H-7500; Hitachi, Tokyo, Japan). The samples were prepared by adding a drop of micelle solution onto 200-mesh carbon-coated copper grids followed by negative staining with $1 \%$ phosphotungstic acid at $\mathrm{pH}$ 7.0. Excess liquid on the copper grids was removed with filter papers and dried in a desiccator until examination.

\section{Critical micelle concentration}

The critical micelle concentration (CMC) was determined using the fluorescent probe pyrene. ${ }^{23}$ The saturated stock pyrene solution was prepared by dissolving pyrene powder in THF. The solution was then added drop-wise into deionized water (oil-in-water method) and the residual THF was removed by evaporation at room temperature overnight. Various concentrations of aqueous polymer solution, ranging from $1 \mathrm{mg} / \mathrm{mL}$ to $10^{-8} \mathrm{mg} / \mathrm{mL}$ (one-tenth dilutions), were prepared using this method. The fluorescence intensity of the micelles was determined for excitation at $310 \mathrm{~nm}$ and scanned emission at $321-450 \mathrm{~nm}$. The ratio of emission intensity I3/I1 (I3, $383 \mathrm{~nm}$; $\mathrm{I} 1,371 \mathrm{~nm}$ ) was determined and used to calculate the CMC.

\section{Lower critical solution temperature behavior}

The lower critical solution temperature (LCST) is defined as the temperature producing half of the total increase in optical absorbance. ${ }^{24}$ The optical absorbance of the empty micelle solution $(1 \mathrm{~g} / \mathrm{L})$ was measured at $500 \mathrm{~nm}$ by ELISA microplate reader (SpectraMax M2 Multi-mode Microplate Reader, Molecular Devices). Sample cells were heated slowly in a circulator bath from $25^{\circ} \mathrm{C}$ to $55^{\circ} \mathrm{C}$ prior to the measurement.

\section{In vitro profile of I7-AAG release from the micelles as a function of temperature}

The profile of 17-AAG release from the micelles was determined by the dialysis bag diffusion method using dialysis membrane with a molecular weight (MW) cutoff of $3.5 \mathrm{kDa}^{2}$ A known volume of IR-780/17-AAG-coloaded micelles was added to a dialysis bag with Tween $80(0.5 \%-1 \%$ total volume) as a surfactant. The bag was then immersed in PBS $(100 \mathrm{~mL})$ and stirred gently at room temperature. The aqueous solution $(100 \mu \mathrm{L})$ was then withdrawn from the release buffer and the absorbance was measured using an ELISA microplate reader.

\section{Cell culture and in vitro cytotoxicity}

The colon cancer cell line (HCT-116) was used for in vitro studies. HCT-116 cells were cultivated in McCoy's 5A modified medium (Sigma-Aldrich Co.) with 10\% (v/v) heat-inactivated fetal bovine serum (Thermo Fisher Scientific, Waltham, MA, USA), $1 \%$ (v/v) penicillin-streptomycin-amphotericin B antibiotic-antimycotic solutions (Sigma-Aldrich Co.) at $37^{\circ} \mathrm{C}$ in an atmosphere of 5\% $\mathrm{CO}_{2}$ humidified incubator. McCoy's $5 \mathrm{~A}$ modified medium was used with further additions of 4 mM L-glutamine (Thermo Fisher Scientific). In vitro cytotoxicity was assayed using 3-(4,5-dimethylthiazol2-yl)-2,5-diphenyltetrazolium bromide (MTT; Alfa Aesar). HCT -116 cells were seeded at a density of 15,000 cells/ well in a 96-well plate and incubated overnight. The culture medium was then replaced with empty micelles, 17-AAG- or IR-780-loaded micelles, or IR-780 and 17-AAG-coloaded micelles. After incubating for 24 hours, MTT solution $(100 \mu \mathrm{L}, 0.4 \mathrm{mg} / \mathrm{mL})$ was added to each well and incubated for 4 hours at $37^{\circ} \mathrm{C} .{ }^{25}$ The culture medium was then 
withdrawn and replaced with DMSO $(100 \mu \mathrm{L})$ to dissolve the purple formazan crystals. The plate was read with an ELISA reader (Sunrise-Basic, Tecan, Switzerland) at a wavelength of $570 \mathrm{~nm}$ and a reference at $630 \mathrm{~nm}$. The percentage of viable cells was calculated as follows: (optical density [OD] of sample/OD of control) $\times 100$.

\section{Temperature measurements and photothermal ablation toxicity}

An $808 \mathrm{~nm}$ laser diode has $0.8 \mathrm{~W} / \mathrm{cm}^{2}$ output power with a center wavelength of $808 \mathrm{~nm}$. To evaluate the change in temperature induced by this laser, we used the infrared thermographic camera (F30s, NEC Avio Infrared Technologies Co., Ltd., Tokyo, Japan) to record the samples per 15 seconds. HCT-116 cells were cultured in 96-well plates with IR-780-loaded micelles, 17-AAG-loaded micelles, or IR-780/17-AAG-coloaded micelles for 5 hours. Cells were then irradiated for 5 minutes and the medium was replaced with fresh medium $(100 \mu \mathrm{L})$. Cells were incubated for 24 hours and cell viability was evaluated by MTT assay.

\section{In vivo antitumor effects of IR-780, I7-AAG, or IR-780/I7-AAG-loaded micelles}

The in vivo experimental protocols were approved by the National Taiwan University College of Medicine and College of Public Health Institutional Animal Care and Use Committee, and the Animal Research: Reporting of In Vivo Experiments guidelines were followed. Scid mice (5-6 weeks old) were purchased from the National Laboratory Animal Center, Taiwan. The mouse colorectal carcinoma (HCT-116) tumor model was established by the subcutaneous injection of $2 \times 10^{6}$ HCT-116 cells. Tumor volumes were calculated as $\mathrm{L} \times \mathrm{W}^{2} / 2$, where $\mathrm{L}$ is the length and $\mathrm{W}$ is the width of the tumor. Mice were treated when the tumors reached a volume of 150-200 $\mathrm{mm}^{3}$, designated as day 1 . Mice were divided into four groups, each receiving different treatments ( $\mathrm{n}=4$ for each group): 1) control, 2) 17-AAG-loaded micelles with laser, 3) IR-780-loaded micelles with laser, and 4) 17-AAG/IR-780-loaded micelles with laser. The scid mice were intravenously injected with one dose of nanoparticles. At 24 hours after nanoparticle injection, tumors were exposed to light from an $808 \mathrm{~nm}$ laser for 2 minutes for photothermal ablation treatment. Tumor sizes and body weights were measured twice weekly during the experiment. ${ }^{26}$

\section{Statistical analysis}

One-way analysis of variance (ANOVA) was used to analyze the differences in the cytotoxicity of 17-AAG-, IR-780-, and
IR-780/17-AAG-loaded micelles. Student's $t$-test was performed to analyze the differences in MTT assays and tumor volumes. $P<0.05$ was considered significant and $P<0.01$ was considered highly significant. The combination index (CI) was determined using CompuSyn software (Combosyn, Inc., Paramus, NJ). The CI provides a quantitative measure of additive effects $(\mathrm{CI}=1)$, synergism $(\mathrm{CI}<1)$, and antagonism $(\mathrm{CI}>1)$ in drug combinations. ${ }^{27}$

\section{Results and discussion Characterization of $P$ (NIPAAM-co- PEGMEA)-b-PCL}

The copolymers comprised the following three components: NIPAAM, PEGMEA, and PCL. NIPAAM provides the copolymers with thermosensitivity, allowing for controlled drug release. PEGMEA protects the micelle from macrophages. The PCL moiety is a hydrophobic segment used to encapsulate the hydrophobic anticancer drug (17-AAG) or photosensitizer (IR-780) through hydrophobic-hydrophobic interactions. Macro-CPPA synthesized by ring opening polymerization and p(NIPAAMco-PEGMEA)-PCL synthesized by RAFT polymerization were characterized by ${ }^{1} \mathrm{H}-\mathrm{NMR}$. The resonances characteristic of PCL $\left(\delta \mathrm{H}^{\mathrm{a}}, 4.05 \mathrm{ppm} ; \delta \mathrm{H}^{\mathrm{b}}, 1.4 \mathrm{ppm} ; \delta \mathrm{H}^{\mathrm{c}}, 2.3 \mathrm{ppm}\right.$; and $\left.\delta \mathrm{H}^{\mathrm{d}}, 1.65 \mathrm{ppm}\right)$, NIPAAM $\left(\delta \mathrm{H}^{\mathrm{e}}, 1.1 \mathrm{ppm}\right)$, and PEGMEA $\left(\delta \mathrm{H}^{\mathrm{f}}, 3.6 \mathrm{ppm}\right)$ were observed (Figure S2). Thus, synthesis of macro-CPPA and p(NIPAAM-co-PEGMEA)-PCL was confirmed. ${ }^{28}$

The MWs and PDIs of macro-CPPA and p(NIPAAM-coPEGMEA)-PCL were determined by GPC, indicating a sharp peak and small PDI of 1.042 and 1.478 for macro-CPPA and $\mathrm{p}$ (NIPAAM-co-PEGMEA)-PCL, respectively. The number average MWs of macro-CPPA and p(NIPAAM-coPEGMEA)-PCL were 6,601 and 16,794 g/mole, respectively. In addition, the left-shifting of the macro-CPPA peak indicated increasing MWs through a series of polymerizations. Therefore, the GPC data demonstrated successful RAFT polymerization (Figure S3), detailed information of which is presented in Table 1.

Table I Molecular weights of polymers (g/mol)

\begin{tabular}{llll}
\hline Sample name & Mn & MW & PDI \\
\hline Macro-CPPA & 6,601 & 6,884 & 1.042 \\
P(NIPAAM-co-PEGMEA)-b-PCL & 16,794 & 24,829 & 1.478 \\
\hline
\end{tabular}

Abbreviations: CPPA, 4-cyano-4-(phenylcarbonothioylthio) pentanoic acid; NIPAAM, N-isopropylacrylamide; PEGMEA, poly(ethylene glycol) methyl ether acrylate; PCL, poly(epsilon-caprolactone); PDI, polydispersity index; MW, molecular weight; Mn, number average MWs. 


\section{Micelle characterization}

Low CMC values are essential to the stability of the micelles upon dilution in the blood. The CMC of empty micelles was found to be $\sim 9 \mathrm{mg} / \mathrm{L}$. This low CMC value indicated that the new polymer p(NIPAAM-co-PEGMEA)-b-PCL formed empty micelles that remained intact when diluted in blood environments (Figure S4).

Table 2 shows the average size, zeta potential, PDIs, and EE of micelles. The particle size and zeta potential of empty micelles were $\sim 91.4 \pm 0.8 \mathrm{~nm}$ (PDI, 0.086) and $-12.9 \pm 0.57 \mathrm{mV}$, respectively. Upon incorporation of 17-AAG or IR-780 into the micelles, the particle size increased to $118.1 \pm 1.5 \mathrm{~nm}$ (PDI, 0.109) and $149.1 \pm 3.2 \mathrm{~nm}$ (PDI, 0.135), respectively. Table 2 shows the size distribution of particles incorporating each drug. The majority of particles were below $0.2 \mathrm{~nm}$ in size, demonstrating that the solutions were homogeneous with a narrow size distribution. The zeta potentials changed only slightly with incorporation of 17-AAG or IR-780(17-AAG alone, $-17.2 \pm 1.92 \mathrm{mV}$; IR-780 alone, $-6.3 \pm 1.12 \mathrm{mV}$; 17-AAG/IR-780-coloading, $-4.52 \pm 0.6 \mathrm{mV}$ ) (Figure S5). These results indicate that loading either or both of the drugs into the micelle core does not affect the zeta potential.

The drug EE of 17-AAG or IR-780 by the micelles was $\sim 51 \%$ and $70 \%$, respectively. Because of the smaller space available to each drug, the EE of dual drug (IR-780/17-AAG)coloaded micelles was $\sim 33 \% / 32 \%$. Transmission electron microscopy (TEM) demonstrated that coloaded micelles were round and of a uniform size (range, 130-200 nm) (Figure 3). Similar particle sizes were indicated by TEM and DLS. The small size of the micelles $(<200 \mathrm{~nm})$ conforms to that required for use in drug delivery system, conferring enhanced permeability and retention effects to increase accumulation in tumors.

Table 2 Characterization and drug load of P(NIPAAM-coPEGMEA)-b-PCL micelles

\begin{tabular}{lllll}
\hline Sample name & Size $(\mathbf{n m})$ & PDI & Zeta $(\mathbf{m V})$ & EE (\%) \\
\hline Empty micelle & $9 I .4 \pm 0.8$ & 0.086 & $-12.9 \pm 0.57$ & - \\
I7-AAG-loaded micelle & $\mathrm{I} 18.1 \pm 1.5$ & 0.109 & $-17.2 \pm 1.92$ & $5 \mathrm{I}$ \\
IR-780-loaded micelle & $\mid 49.1 \pm 3.2$ & 0.135 & $-6.3 \pm 1.12$ & 70 \\
IR-780/I7-AAG- & $\mid 75.5 \pm 4.1$ & 0.167 & $-4.52 \pm 0.6$ & $33 / 32$ \\
coloaded micelle & & & & \\
\hline
\end{tabular}

Notes: Weight ratio $=$ weight ratio of drug to p(NIPAAM-co-PEGMEA)-b-PCL; EE (encapsulation efficiency) = weight of drug in micelles/weight of total drug $\times 100 \%$.

Abbreviations: NIPAAM, N-isopropylacrylamide; PEGMEA, poly(ethylene glycol) methyl ether acrylate; PCL, poly(epsilon-caprolactone); 17-AAG, 17-allylamino-17demethoxygeldanamycin; IR, infrared.

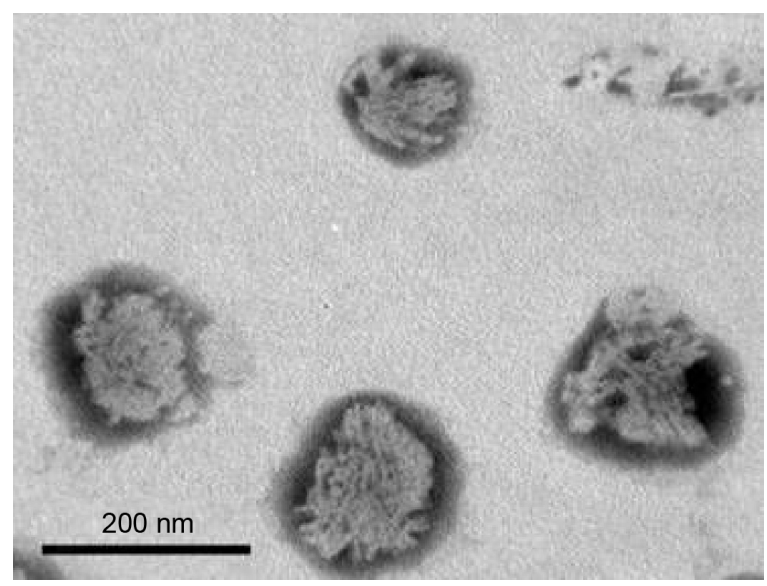

Figure 3 TEM images of IR-780/I7-AAG-coloaded micelles.

Abbreviations: TEM, transmission electron microscopy; I7-AAG, 17-allylamino17-demethoxygeldanamycin; IR, infrared.

The thermosensitive polymer in the micelles triggered a hydration-dehydration change in the micelles in an aqueous solution when the environmental temperature was close to the LCST. This change disrupted the conformation of the micelles, releasing the drug. In addition, the hydroxyl group of PEGMEA enhanced the hydrophilicity and modulated the LCST of the p(NIPAAM-co-PEGMEA)-b-PCL copolymer. The LCST of empty micelles was $43^{\circ} \mathrm{C}$ (Figure S6). This characteristic may facilitate drug release at higher temperatures, conferring control over drug release.

\section{Drug release profiles}

Because of the photobleaching effect, the concentration of IR-780 in the micelles could not be accurately measured after irradiation. The release profile of 17-AAG in IR-780/17-AAGcoloaded micelles with or without laser treatment was carried out by dialysis bag diffusion method at $37^{\circ} \mathrm{C}$ in PBS and the results are shown in Figure 4. The IR-780/17-AAG-coloaded micelles without laser treatment released only $50.1 \%$ of the 17-AAG contents in 24 hours. With laser treatment, rapid 17-AAG release was observed after 5 minutes. The fraction of 17 -AAG released was $\sim 53 \%$ after 5 hours and $\sim 65 \%$ after 24 hours. When IR-780/17-AAG-coloaded micelles were irradiated by laser, the photothermal effect of IR-780 heated the environment. Then, the thermosensitive polymeric micelle became unstable and facilitated more drug release. Because the polymer of NIPAAM with LCST causes a coil-globule transition of polymer chains, the micelle composed of copolymer, p(NIPAAM-co-PEGMEA)-b-PCL, shows decreased swelling levels with increasing temperatures and deswelling dramatically at the temperature near LCST. This mechanism can also increase 


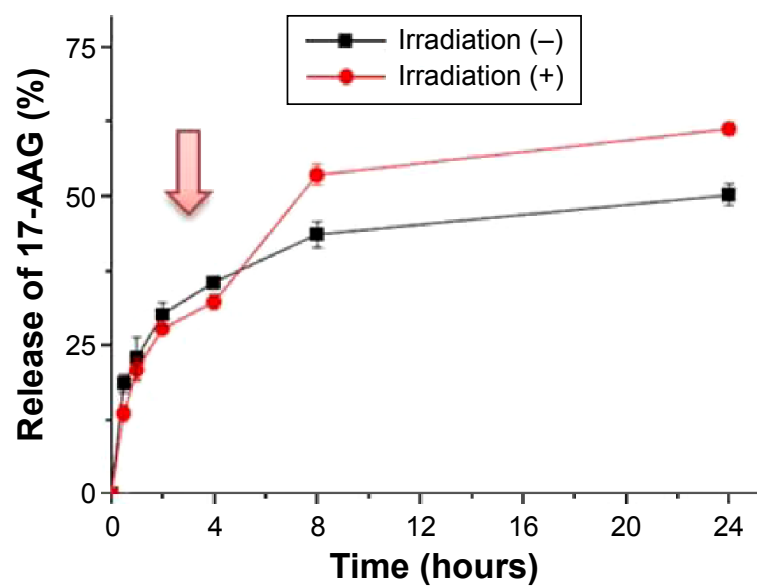

Figure 4 Drug release profiles of IR-780/I7-AAG-coloaded micelles in PBS at $37^{\circ} \mathrm{C}$.

Note: Red arrow indicates the time point of light treatment.

Abbreviations: I7-AAG, I7-allylamino-17-demethoxygeldanamycin; IR, infrared; PBS, phosphate buffered saline.

the amount of drug release. Moreover, because of the destruction of hydrophilic and hydrophobic balance of the IR-780/17AAG-coloaded micelles, the data showed that the drug release behavior of the micelles was temperature-dependant. Thus, the micelles exhibited thermosensitive release of the drug. In addition, the increased release of 17-AAG may improve the inhibition of HSP-90.

\section{In vitro photothermal property of IR-780/I 7-AAG micelles}

The infrared-absorbing property of IR-780 ( $\left.\mathrm{A}_{\max }, 800 \mathrm{~nm}\right)$ was used to create photosensitive micelles for use in photothermal ablation therapy. Near-infrared (NIR) light with IR-780 converts light of the appropriate wavelength into heat, killing cancer cells. Only IR-780-loaded micelles promoted an increase in the temperature of the environment in response to light (Figure 5A). Within 5 minutes of laser irradiation, the solution of IR-780/17-AAG-coloaded micelles reached $\sim 55^{\circ} \mathrm{C}$, whereas that of micelles containing 17-AAG alone remained at $30^{\circ} \mathrm{C}$ (Figure 5B). This inducible temperature increase could be used to release the drug during photothermal ablation therapy. Previous studies have reported that photothermal ablation therapy requires the temperature to increase above $43^{\circ} \mathrm{C}$, which was clearly achieved by these micelles. ${ }^{9}$

\section{In vitro photothermal toxicity and synergistic effects}

The viability of HCT-116 cells treated with empty p(NIPAAM-co-PEGMEA)-b-PCL micelles is shown in Figure S7. Addition of the nontoxic polymers PEGMEA and PCL does not decrease cell viability at concentrations below $100 \mu \mathrm{g} / \mathrm{mL}$. The viability of cells treated with either $50 \mu \mathrm{g} / \mathrm{mL}$ 17-AAG-loaded micelles or free 17-AAG-loaded micelles was $66.03 \%$ and $57.2 \%$, respectively. Thus, the cytotoxicity of 17-AAG is maintained in the micelles. However, lower cytotoxicity of 17-AAG in micelles loaded with higher concentrations of the drug occurs as a result of encapsulation of the drug in the micelle, decreasing the drug release and facilitating micelle accumulation in the tumor.

HCT-116 cells were treated with IR-780-loaded micelles $(10 \mu \mathrm{g} / \mathrm{mL})$ for 24 hours and excited with an $808 \mathrm{~nm}$ laser diode. Similar effects were observed in IR-780-loaded micelles and free IR-780 without laser irradiation (Figure 6).

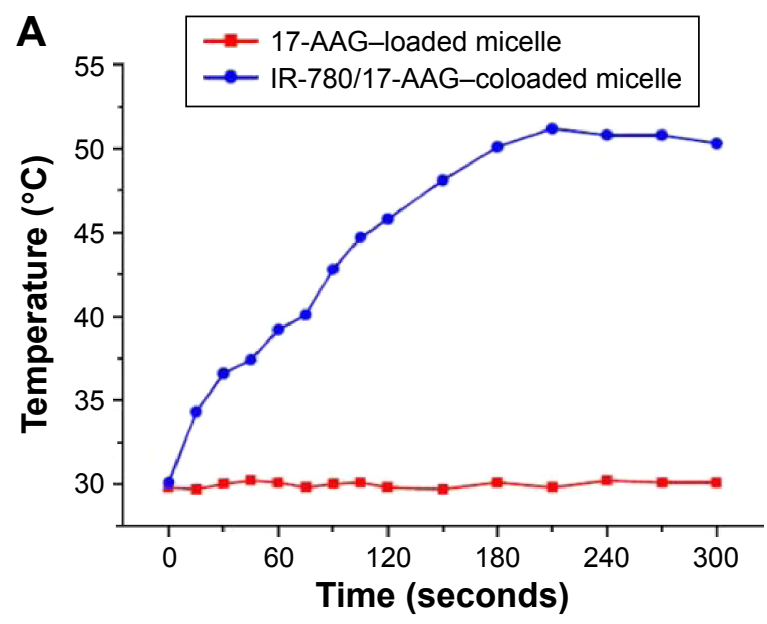

B

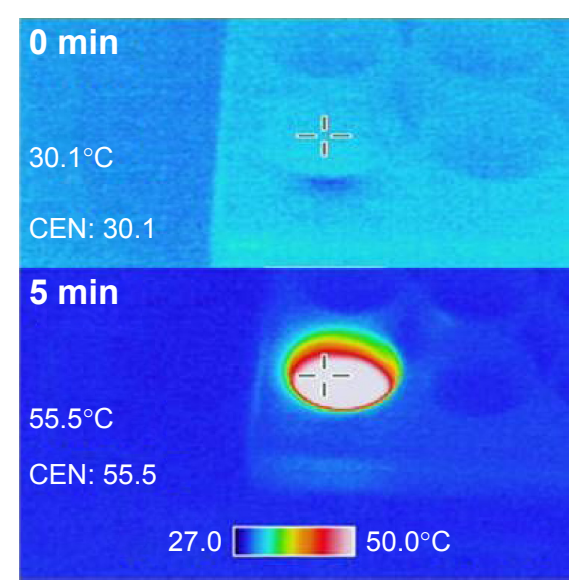

Figure 5 In vitro photothermal property of IR-780/I7-AAG micelles.

Notes: (A) Temperature change in IR-780/I7-AAG-coloaded micelles and I7-AAG-loaded micelles during continuous irradiation. (B) Image of IR-780/I7-AAG-coloaded micelles during continuous irradiation for 5 minutes $\left(808 \mathrm{~nm}\right.$ laser at $\left.0.8 \mathrm{~W} / \mathrm{cm}^{2}\right)$.

Abbreviations: 17-AAG, 17-allylamino-17-demethoxygeldanamycin; IR, infrared; CEN, center. 

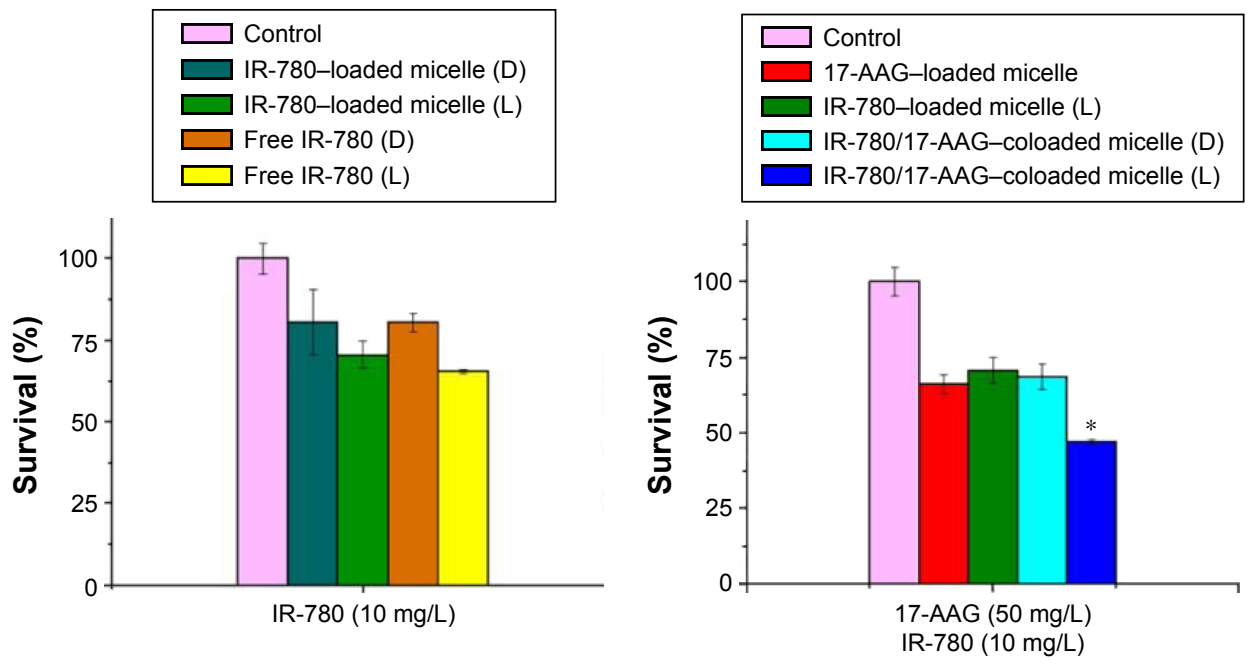

Figure 6 The cytotoxicity at 24 hours of IR-780-loaded micelles and free drug, with or without light irradiation. Note: $* p<0.05$.

Abbreviations: I7-AAG, I7-allylamino-17-demethoxygeldanamycin; IR, infrared; D, without laser; L, with laser.

Cells treated with NIR irradiation exhibited lower survival rate than those treated without light irradiation. These results indicate that IR-780 loaded into micelles is a potential photosensitizer for photothermal ablation therapy.

The goal of this study was to combine chemotherapy and photothermal ablation therapy to create a new delivery system. After 24 hours of incubation, IR-780/17-AAGcoloaded micelles exhibited significant killing of HCT-116 cells upon laser irradiation, with growth inhibition $(>50 \%)$, over that of cells treated with micelles containing either drug alone. IR-780/17-AAG-coloaded micelles had synergistic effects on cell killing, as indicated by the CI values (Table 3 ). The CI value of HCT-116 cells treated with micelles containing $50 \mu \mathrm{g} / \mathrm{mL} 17-A A G$ and $10 \mu \mathrm{g} / \mathrm{mL}$ IR-780 plus laser irradiation was 0.87 . The synergistic effect $(\mathrm{CI}<1)$ was thus verified. These results indicate that with laser irradiation, the raising temperature provided not only controlled release of 17-AAG but also photothermal ablation therapy in vitro.

\section{In vivo antitumor effects of IR-780 alone, I7-AAG alone, or IR-780 plus I7-AAG- loaded micelles upon NIR irradiation}

As shown in Figure 7A, scid mice (5-6 weeks old) were used to establish a colon cancer tumor model; mice were treated when the tumors reached a volume of $150-200 \mathrm{~mm}^{3}$. The antitumor efficacy of 17-AAG-, IR-780-, and IR-780 plus 17-AAG-loaded

Table 3 Combination indexes

\begin{tabular}{lllll}
\hline & I7-AAG $(\mathrm{mg} / \mathrm{L})$ & IR-780 (mg/L) & Cl value & \\
\hline HCT-I I6 & 50 & 10 & 0.87 & synergism \\
\hline
\end{tabular}

Abbreviations: HCT, human colorectal adenocarcinoma; 17-AAG, 17-allylaminoI7-demethoxygeldanamycin; IR, infrared; $\mathrm{Cl}$, combination index. micelles with or without $808 \mathrm{~nm}$ laser $\left(0.8 \mathrm{~W} / \mathrm{cm}^{2}, 2\right.$ minutes $)$ was evaluated in the tumor model. The loss of body weight in mice was used as a measure of treatment effects. None of the mice exhibited significant changes in body weight during the treatment, indicating a potential for clinical use (Figure 7B). In addition, the group treated with empty micelles exhibited no significant therapeutic effect, showing that the empty micelle was well tolerated and exhibited no toxicity in vivo. Figure 7B shows that the tumor size in the control group continues to grow over the course of time. Tumor growth decreased slightly in the group treated with 17-AAG-loaded micelles and in the group treated with IR-780-loaded micelles with laser irradiation. However, mice treated with IR-780/17-AAG-loaded micelles with laser irradiation exhibited significantly greater suppression of tumor growth than did those undergoing single treatment. The increase in temperature at the tumor site after 2 minutes $\left(47.2^{\circ} \mathrm{C}\right.$ ) achieved the effect of PTT (Figure 8). Thus, the use of thermosensitive NIPAAM in micelles improved the release of 17-AAG during the temperature increase. The combination of photothermal ablation therapy and chemotherapy provided better treatment. The in vivo results illustrating the effects of treatment with IR-780/17-AAG-loaded micelles are in accordance with the in vitro results.

\section{Conclusion}

In this study, a thermosensitive material based on p(NIPAAMco-PEGMEA)-b-PCL copolymers was successfully fabricated and self-assembled into micelles that were coloaded with an HSP inhibitor (17-AAG) and a photothermal agent (IR-780). IR-780/17-AAG-coloaded micelles exhibited characteristics consistent with use as a multifunction nanomedicine for dual PTT and chemotherapy. Furthermore, 17-AAG was released 
A

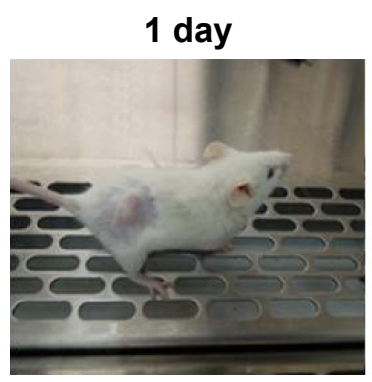

3 days

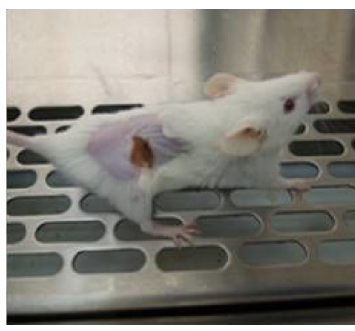

8 days

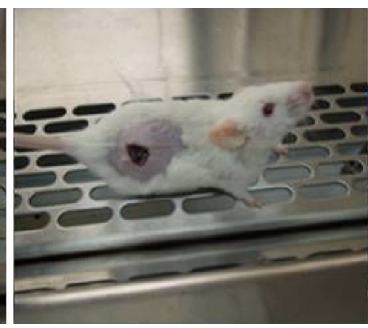

15 days

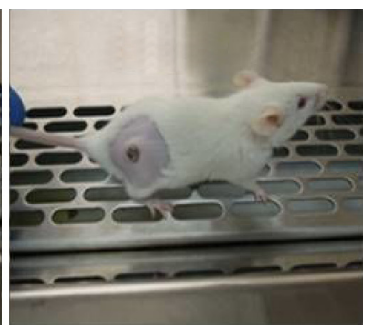

B
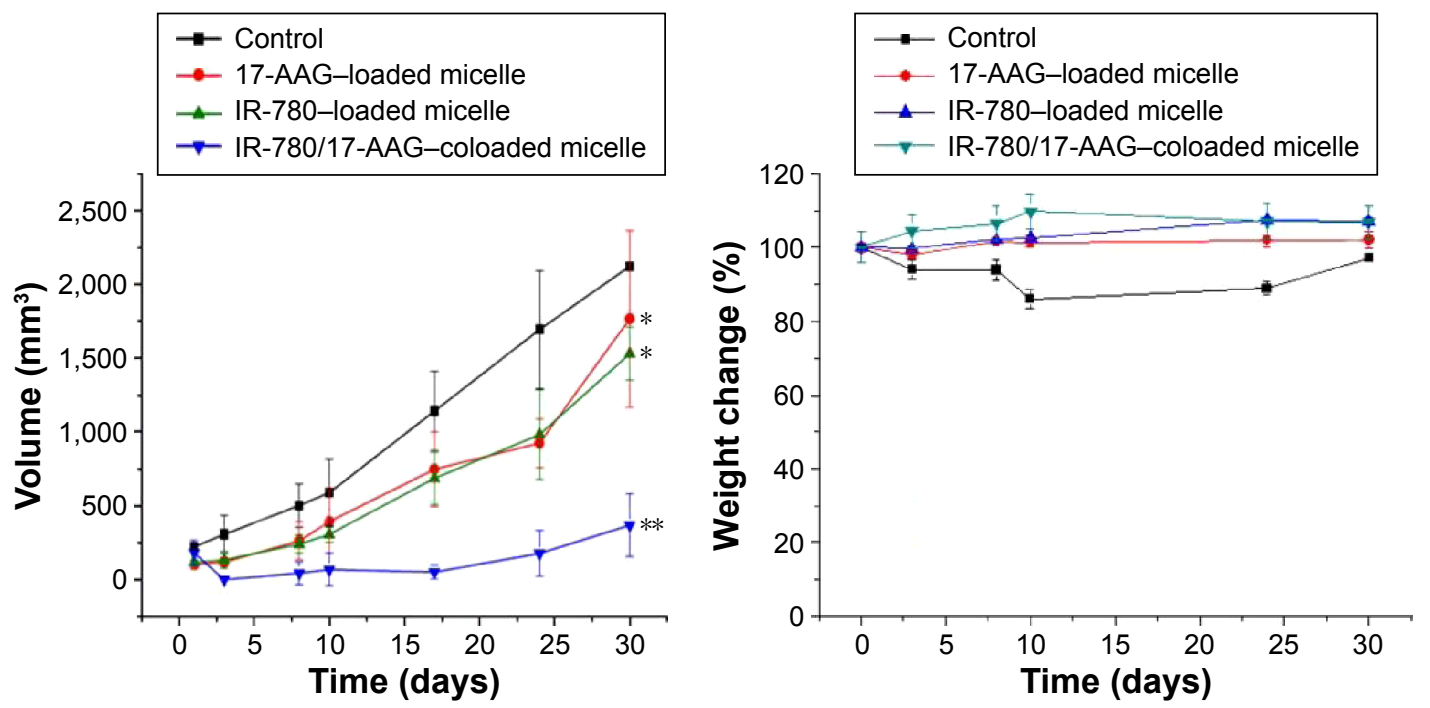

Figure 7 In vivo antitumor effects of IR-780 alone, I7-AAG alone, or IR-780 plus I7-AAG-loaded micelles upon NIR irradiation.

Notes: (A) Photographs of scid mice at the indicated days after treatment with IR-780/I7-AAG-coloaded micelles. (B) Tumor volumes and body weights of scid mice treated with I7-AAG-loaded micelles with NIR irradiation, I7-AAG-loaded micelles alone, or IR-780/I7-AAG-coloaded micelles with NIR irradiation. $* P<0.05$; $* * P<0.0$ I.

Abbreviations: I7-AAG, I7-allylamino-17-demethoxygeldanamycin; IR, infrared; NIR, near-infrared.

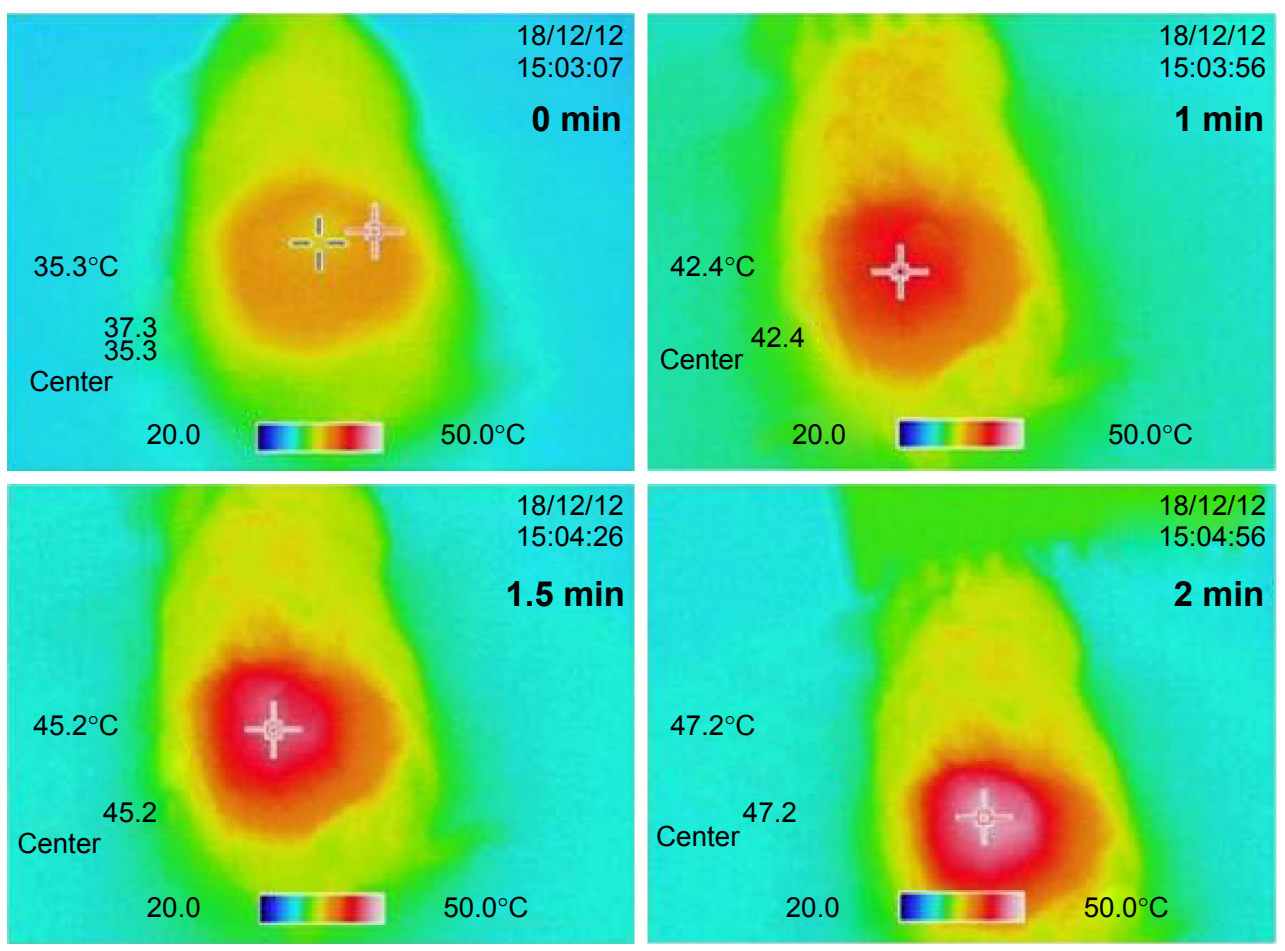

Figure 8 Tumor temperature and infrared thermographic maps of nude mice bearing HCT-II 6 tumors injected with IR-780/I7-AAG-coloaded micelles. Abbreviations: 17-AAG, 17-allylamino-17-demethoxygeldanamycin; IR, infrared. 
more effectively during laser irradiation due to the thermosensitivity of the micelles. The combination of chemotherapy and PTT was synergistic, not only inducing HCT-116 cell death in vitro but also suppressing HCT-116 tumor growth in vivo. These novel thermosensitive p(NIPAAM-co-PEGMEA)-bPCL micelles may be ideal nanocarriers for clinical use to provide controlled delivery of 17-AAG and IR-780.

\section{Acknowledgments}

This research was supported by grants from the National Science Council of the Republic of China (NSC 102-2320B-002-038-MY3, NSC 102-2221-E-002-037-MY3).

\section{Author contributions}

Cheng-Liang Peng: experimental design, performance of experiments, providing suggestions, and manuscript revision; Yuan-I Chen: experimental design and manuscript writing; Hung-Jen Liu: experimental design and performance of experiments; Pei-Chi Lee: participation in the experiment involving animal model; Tsai-Yueh Luo: experimental suggestions; Ming-Jium Shieh: experimental suggestions and manuscript revision. All authors contributed toward data analysis, drafting and critically revising the paper and agree to be accountable for all aspects of the work.

\section{Disclosure}

The authors report no conflicts of interest in this work.

\section{References}

1. Mohamed Hamouda I. Current perspectives of nanoparticles in medical and dental biomaterials. J Biomed Res. 2012;26(3):143-151.

2. Qu T, Wang A, Yuan J, Shi J, Gao Q. Preparation and characterization of thermo-responsive amphiphilic triblock copolymer and its self-assembled micelle for controlled drug release. Colloids Surf B Biointerfaces. 2009; 72(1):94-100.

3. Zhang JX, Wang K, Mao ZF, et al. Application of liposomes in drug development - focus on gastroenterological targets. Int J Nanomedicine. 2013;8:1325-1334.

4. Liu Y, Miyoshi H, Nakamura M. Nanomedicine for drug delivery and imaging: a promising avenue for cancer therapy and diagnosis using targeted functional nanoparticles. Int J Cancer. 2007;120(12):2527-2537.

5. Katragadda U, Teng Q, Rayaprolu BM, Chandran T, Tan C. Multi-drug delivery to tumor cells via micellar nanocarriers. Int J Pharm. 2011; 419(1-2):281-286.

6. Maeda $\mathrm{H}$. The link between infection and cancer: tumor vasculature, free radicals, and drug delivery to tumors via the EPR effect. Cancer Sci. 2013;104(7):779-789.

7. Peng C-L, Shih Y-H, Lee P-C, Hsieh TM-H, Luo T-Y, Shieh M-J. Multimodal image-guided photothermal therapy mediated by Re-188-labeled micelles containing a cyanine-type photosensitizer. Acs Nano. 2011; 5(7):5594-5607.

8. Lim C-K, Shin J, Lee Y-D, et al. Phthalocyanine-aggregated polymeric nanoparticles as tumor-homing near-infrared absorbers for photothermal therapy of cancer. Theranostics. 2012;2(9):871-879.

9. Chen YI, Peng CL, Lee PC, et al. Traceable self-assembly of lasertriggered cyanine-based micelle for synergistic therapeutic effect. Adv Healthc Mater. 2015;4(6):892-902.
10. Rylander MN, Feng Y, Bass J, Diller KR. Thermally induced injury and heat-shock protein expression in cells and tissues. Ann N Y Acad Sci. 2005;1066:222-242.

11. Cheung CH, Chen HH, Cheng LT, Lyu KW, Kanwar JR, Chang JY. Targeting Hsp90 with small molecule inhibitors induces the overexpression of the anti-apoptotic molecule, survivin, in human A549, HONE-1 and HT-29 cancer cells. Mol Cancer. 2010;9:77.

12. Banerji U, O’Donnell A, Scurr M, et al. Phase I pharmacokinetic and pharmacodynamic study of 17-allylamino, 17-demethoxygeldanamycin in patients with advanced malignancies. J Clin Oncol. 2005;23(18): 4152-4161.

13. Peng CL, Lai PS, Lin FH, Yueh-Hsiu Wu S, Shieh MJ. Dual chemotherapy and photodynamic therapy in an HT-29 human colon cancer xenograft model using SN-38-loaded chlorin-core star block copolymer micelles. Biomaterials. 2009;30(21):3614-3625.

14. Shin HC, Cho H, Lai TC, Kozak KR, Kolesar JM, Kwon GS. Pharmacokinetic study of 3-in-1 poly(ethylene glycol)-block-poly(D, L-lactic acid) micelles carrying paclitaxel, 17-allylamino-17-demethoxygeldanamycin, and rapamycin. J Control Release. 2012;163(1):93-99.

15. Hasenstein JR, Shin HC, Kasmerchak K, Buehler D, Kwon GS, Kozak KR. Antitumor activity of Triolimus: a novel multidrug-loaded micelle containing Paclitaxel, Rapamycin, and 17-AAG. Mol Cancer Ther. 2012; 11(10):2233-2242.

16. Huang HC, Yang Y, Nanda A, Koria P, Rege K. Synergistic administration of photothermal therapy and chemotherapy to cancer cells using polypeptide-based degradable plasmonic matrices. Nanomedicine (Lond). 2011;6(3):459-473.

17. Saxena V, Hussain MD. Formulation and in vitro evaluation of 17-allyamino-17-demethoxygeldanamycin (17-AAG) loaded polymeric mixed micelles for glioblastoma multiforme. Colloids Surf B Biointerfaces. 2013;112:350-355.

18. Chang C, Wei H, Quan C-Y, et al. Fabrication of thermosensitive PCLPNIPAAm-PCL triblock copolymeric micelles for drug delivery. J Poly Sci Part A: Polym Chem. 2008;46(9):3048-3057.

19. Li YY, Zhang XZ, Zhu JL, Cheng H, Cheng SX, Zhuo RX. Selfassembled, thermoresponsive micelles based on triblock PMMA-bPNIPAAm-b-PMMA copolymer for drug delivery. Nanotechnology. 2007;18(21):215605.

20. Krimmer SG, Pan H, Liu J, Yang J, Kopecek J. Synthesis and characterization of poly(epsilon-caprolactone)-block-poly[N-(2-hydroxypropyl) methacrylamide] micelles for drug delivery. Macromol Biosci. 2011; 11(8):1041-1051.

21. Boyer C, Boutevin G, Robin JJ, Boutevin B. Study of the telomerization of dimethylaminoethyl methacrylate (DMAEMA) with mercaptoethanol. Application to the synthesis of a new macromonomer. Polymer. 2004;45(23):7863-7876.

22. Egger H, McGrath KM. Aging of oil-in-water emulsions: the role of the oil. J Colloid Interface Sci. 2006;299(2):890-899.

23. Basu Ray G, Chakraborty I, Moulik SP. Pyrene absorption can be a convenient method for probing critical micellar concentration $(\mathrm{cmc})$ and indexing micellar polarity. J Colloid Interface Sci. 2006;294(1): 248-254.

24. Liu Y, Li C, Wang HY, Zhang XZ, Zhuo RX. Synthesis of thermoand $\mathrm{pH}$-sensitive polyion complex micelles for fluorescent imaging. Chemistry. 2012;18(8):2297-2304.

25. Mosmann T. Rapid colorimetric assay for cellular growth and survivalapplication to proliferation and cyto-toxicity assays. J Immunol Methods. 1983;65(1-2):55-63.

26. Katragadda U, Fan W, Wang Y, Teng Q, Tan C. Combined delivery of paclitaxel and tanespimycin via micellar nanocarriers: pharmacokinetics, efficacy and metabolomic analysis. PloS one. 2013;8(3):e58619.

27. Chou TC. Drug combination studies and their synergy quantification using the Chou-Talalay method. Cancer Res. 2010;70(2):440-446.

28. Guo S, Qiao Y, Wang W, et al. Synthesis and properties of Polycaprolactone-graft-poly(2-(dimethylamino)ethyl methacrylateco-methoxy polyethylene glycol monomethacrylate) as non-viral gene vector. Polym Advan Technol. 2011;22(12):1925-1930. 


\section{Supplementary materials}
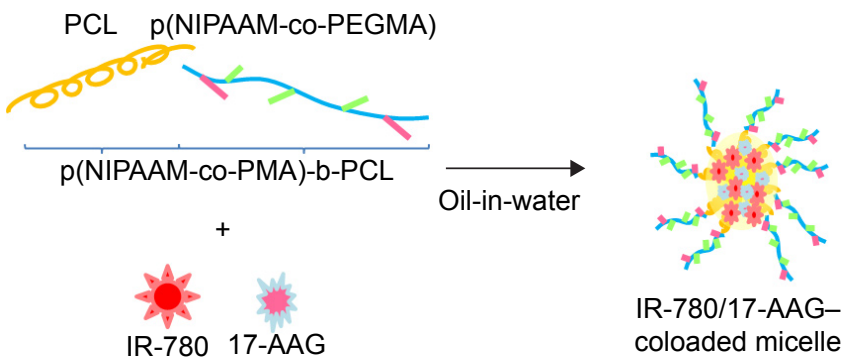

IR-780/17-AAG-

coloaded micelle

Figure SI Preparation of IR-780/I7-AAG-coloaded micelles.

Abbreviations: NIPAAM, N-isopropylacrylamide; PEGMEA, poly(ethylene glycol) methyl ether acrylate; PCL, poly(epsilon-caprolactone); 17-AAG, 17-allylamino-17demethoxygeldanamycin; IR, infrared.

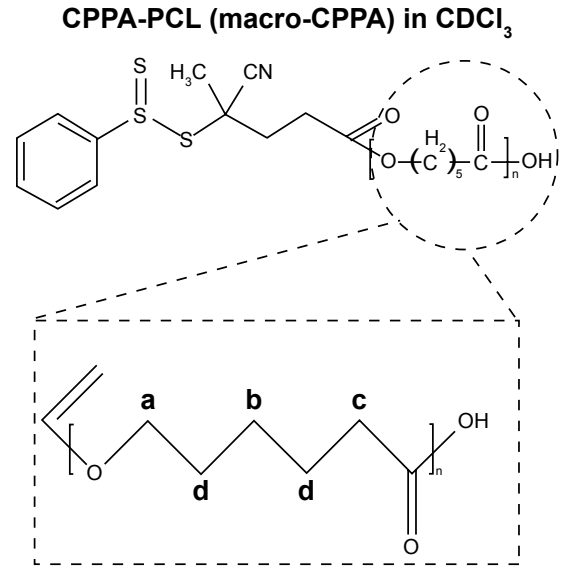

PCL
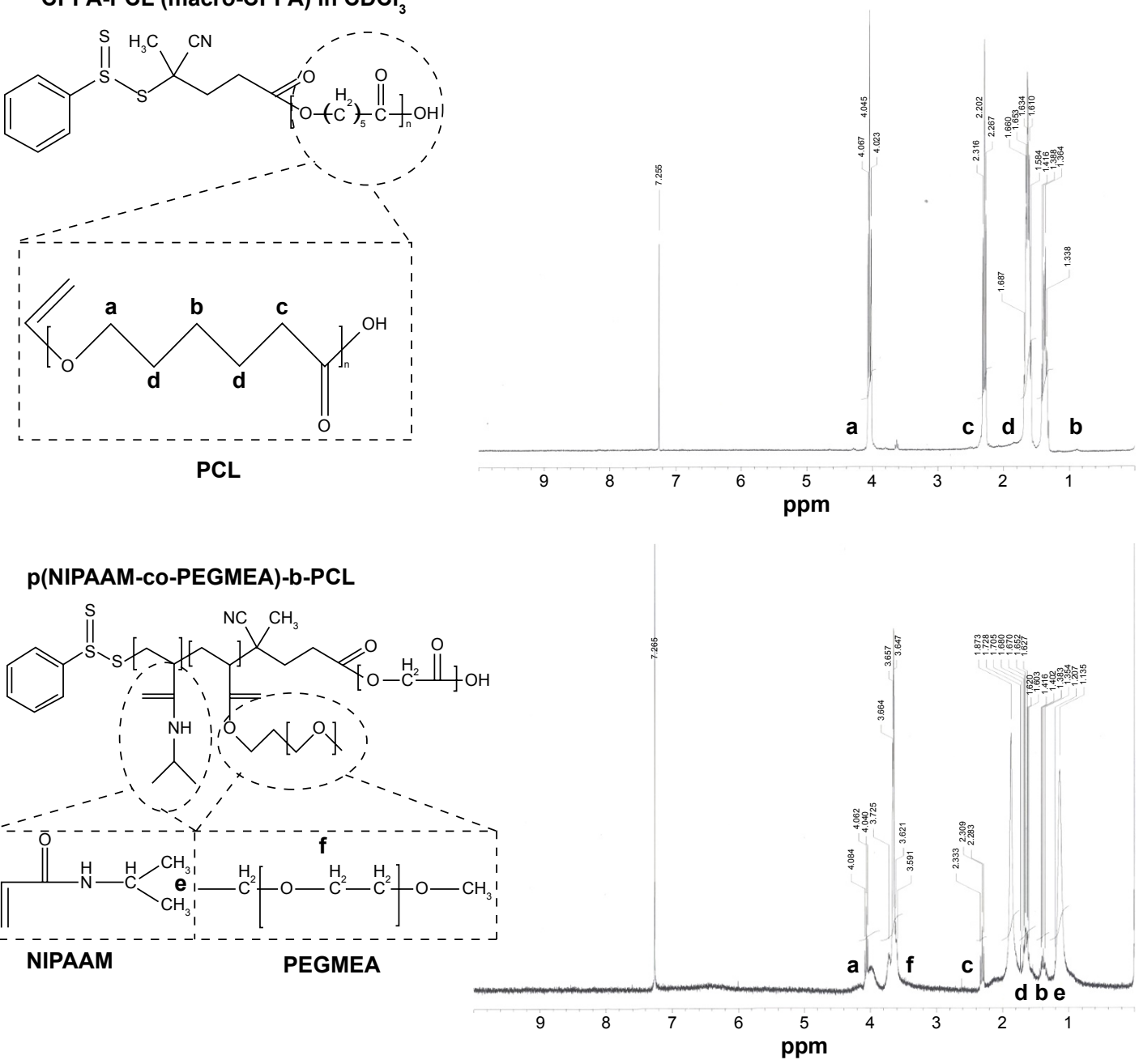

Figure $\mathbf{S 2}$ 'H NMR spectra of CPPA-PCL (macro-CPPA) and P(NIPAAM-co-PEGMEA)-b-PCL in CDCl.

Note: The a-f correspond with the left chemical structure and they can also be noted on the chemical structure $(\delta \mathrm{Ha}, 4.05 \mathrm{ppm} ; \delta \mathrm{Hb}, \mathrm{I} .4 \mathrm{ppm} ; \delta \mathrm{Hc}, 2.3 \mathrm{ppm} ;$ and $\delta \mathrm{Hd}$, I.65 ppm), NIPAAM ( $\delta \mathrm{He}, \mathrm{l} . \mathrm{I} \mathrm{ppm})$, and PEGMEA ( $\delta \mathrm{Hf}, 3.6 \mathrm{ppm})$.

Abbreviations: NMR, nuclear magnetic resonance; CPPA, 4-cyano-4-(phenylcarbonothioylthio) pentanoic acid; PCL, poly(epsilon-caprolactone); NIPAAM, $\mathrm{N}$-isopropylacrylamide; PEGMEA, poly(ethylene glycol) methyl ether acrylate; $\mathrm{CDCl}_{3}$, deuterated chloroform. 


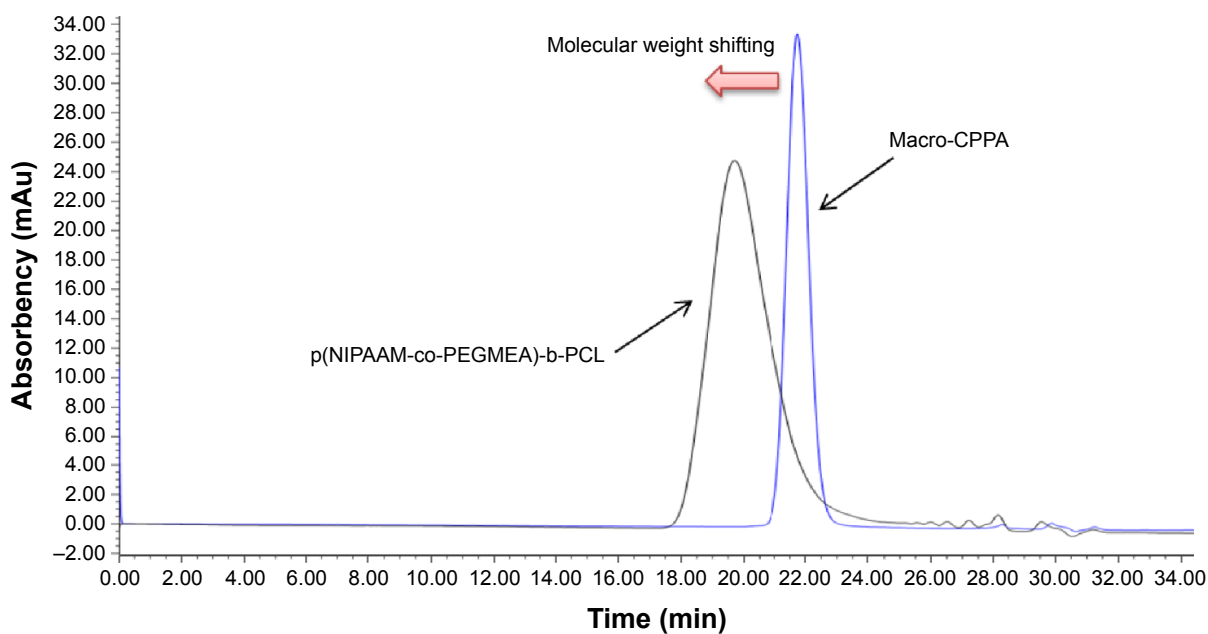

Figure S3 GPC spectrum of macro-CPPA and p(NIPAAM-co-PEGMEA)-b-PCL copolymers.

Abbreviations: GPC, gel permeation chromatography; CPPA, 4-cyano-4-(phenylcarbonothioylthio) pentanoic acid; PCL, poly(epsilon-caprolactone); NIPAAM, $\mathrm{N}$-isopropylacrylamide; PEGMEA, poly(ethylene glycol) methyl ether acrylate; $\mathrm{MW}$, molecular weight.

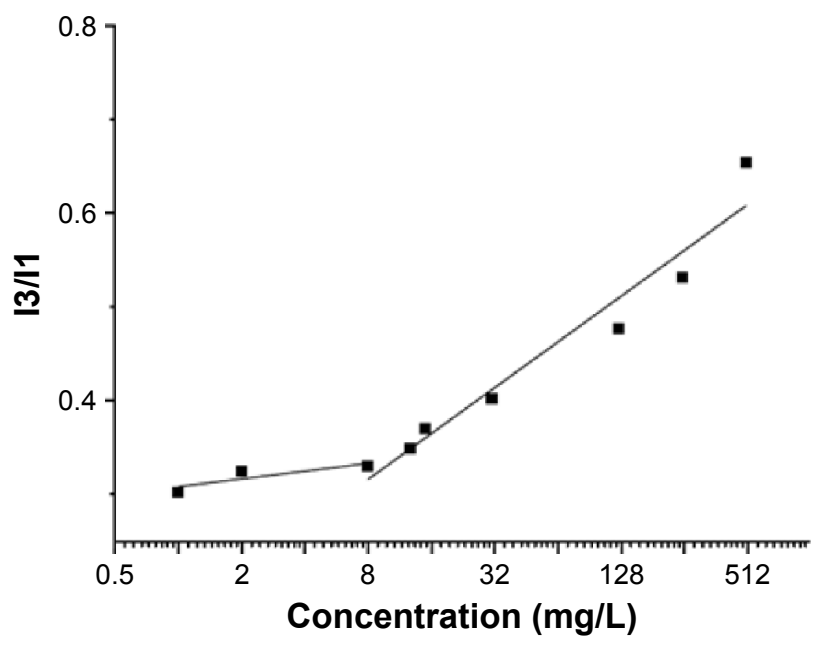

Figure S4 Plot to determine the critical micelle concentration.

Abbreviations: 13 , emission intensity at $383 \mathrm{~nm}$; II, emission intensity at $37 \mathrm{Imm}$.

A

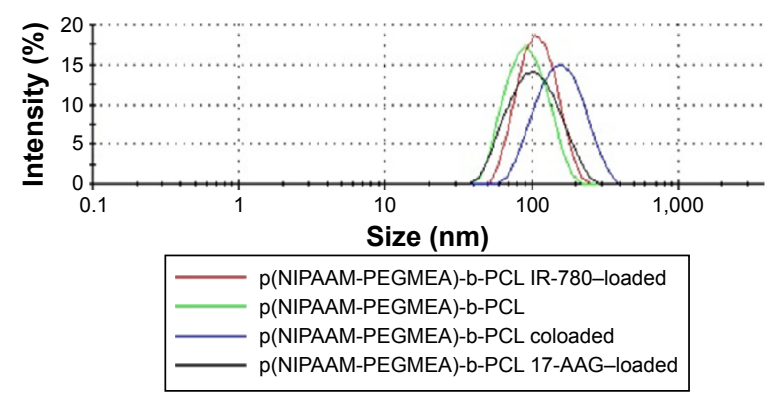

B

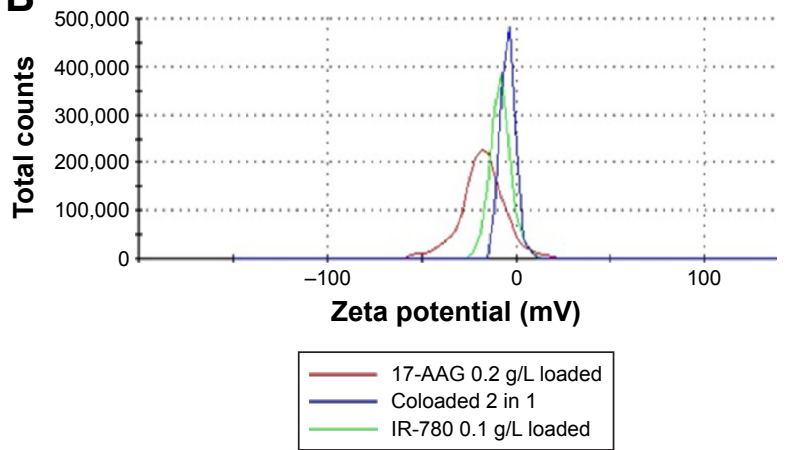

Figure S5 Dynamic light scattering to determine micelle size $(\mathbf{A})$ and zeta potential (B).

Abbreviations: PCL, poly(epsilon-caprolactone); NIPAAM, N-isopropylacrylamide; PEGMEA, poly(ethylene glycol) methyl ether acrylate; I7-AAG, I7-allylamino-I7demethoxygeldanamycin; IR, infrared. 


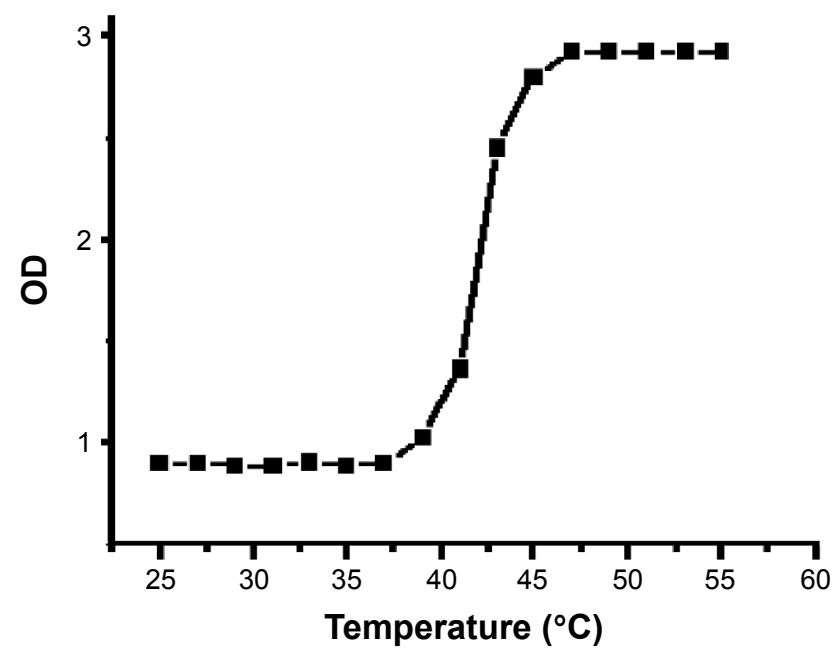

Figure S6 Absorbance curve of empty micelle solution as a function of temperature. Abbreviation: OD, optical density.
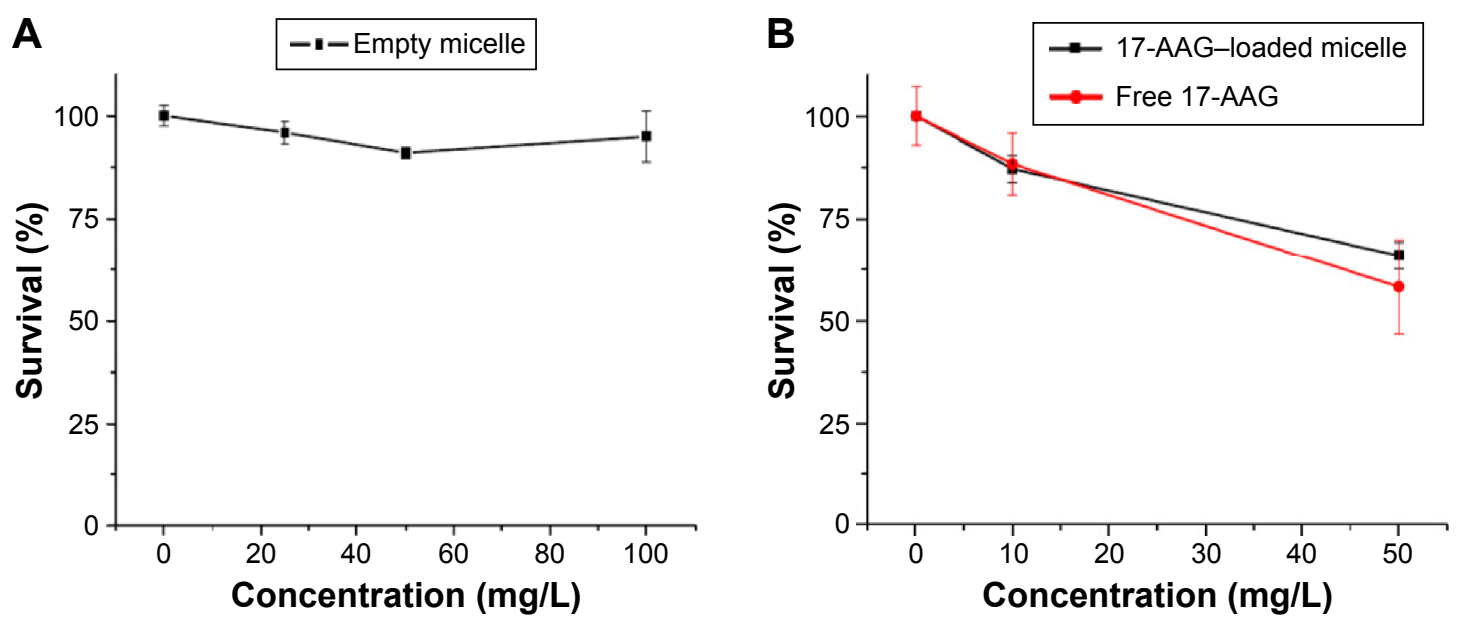

Figure $\mathbf{S 7}$ The viability of HCT- I I 6 cells treated with micelles.

Notes: (A) The cytotoxicity of empty micelles on HCT-II6 cells after 24 hours. (B) The cytotoxicity of free 17-AAG-and I7-AAG-loaded micelles on HCT-II6 cells after 24 hours.

Abbreviations: I7-AAG, 17-allylamino-17-demethoxygeldanamycin; HCT, human colorectal adenocarcinoma.

International Journal of Nanomedicine

\section{Publish your work in this journal}

The International Journal of Nanomedicine is an international, peerreviewed journal focusing on the application of nanotechnology in diagnostics, therapeutics, and drug delivery systems throughou the biomedical field. This journal is indexed on PubMed Central, MedLine, CAS, SciSearch ${ }^{\circledR}$, Current Contents ${ }^{\circledR} /$ Clinical Medicine,

\section{Dovepress}

Journal Citation Reports/Science Edition, EMBase, Scopus and the Elsevier Bibliographic databases. The manuscript management system is completely online and includes a very quick and fair peer-review system, which is all easy to use. Visit http://www.dovepress.com/ testimonials.php to read real quotes from published authors. 